\title{
Thermal stability of the krypton Hall effect thruster*
}

\author{
Agnieszka Szelecka, \\ Jacek Kurzyna, \\ Loic Bourdain
}

\begin{abstract}
The Krypton Large IMpulse Thruster (KLIMT) ESA/PECS project, which has been implemented in the Institute of Plasma Physics and Laser Microfusion (IPPLM) and now is approaching its final phase, was aimed at incremental development of a $\sim 500 \mathrm{~W}$ class Hall effect thruster (HET). Xenon, predominantly used as a propellant in the state-of-the-art HETs, is extremely expensive. Krypton has been considered as a cheaper alternative since more than fifteen years; however, to the best knowledge of the authors, there has not been a HET model especially designed for this noble gas. To address this issue, KLIMT has been geared towards operation primarily with krypton. During the project, three subsequent prototype versions of the thruster were designed, manufactured and tested, aimed at gradual improvement of each next exemplar. In the current paper, the heat loads in new engine have been discussed. It has been shown that thermal equilibrium of the thruster is gained within the safety limits of the materials used. Extensive testing with both gases was performed to compare KLIMT's thermal behaviour when supplied with krypton and xenon propellants.
\end{abstract}

Keywords: thermal stability $\bullet$ electric propulsion $\bullet$ Hall thruster $\bullet$ xenon and krypton propellants

\author{
A. Szelecka ${ }^{\bowtie}$, J. Kurzyna \\ Institute of Plasma Physics and Laser Microfusion, \\ 23 Hery Str., 01-497 Warsaw, Poland, \\ E-mail: agnieszka.szelecka@ifpilm.pl \\ L. Bourdain \\ Université d'Orléans, France
}

Received: 31 August 2016

Accepted: 19 December 2016

\section{Introduction}

Hall effect thruster (HET) is a plasma accelerator with magnetized electrons in which ions that produce the thrust are sped-up by electric field $\mathbf{E}$ that is maintained in the discharge volume owing to the presence of perpendicular magnetic field $\mathbf{B}$ [1].

Since the first launch of a HET flight-model at the Russian satellite of Meteor series [2] the electric propulsion (EP) has well established its competitive position with respect to the classic rocket engines of combustion and cold gas type. Due to the several times higher specific impulse generated by most EP technologies, the mass budget of a small spacecraft could be optimized leaving more room for the mission payload. HETs have already proved their superior capabilities when applied for station keeping or orbit rising, and are more and more frequently being considered as the main engines for future deep space missions. A worthy example that illustrates such a task feasibility is the Small Mission for Advanced Research in Technology 1 (SMART-1) ESA project, which had the purpose to test several new techniques, as well as provide for the observation of lunar surface. The SMART-1 satellite was equipped

\footnotetext{
*This paper is based on a presentation given at 13th Kudowa Summer School "Towards Fusion Energy", Kudowa Zdrój, Poland, 13-17 June 2016.
} 
a

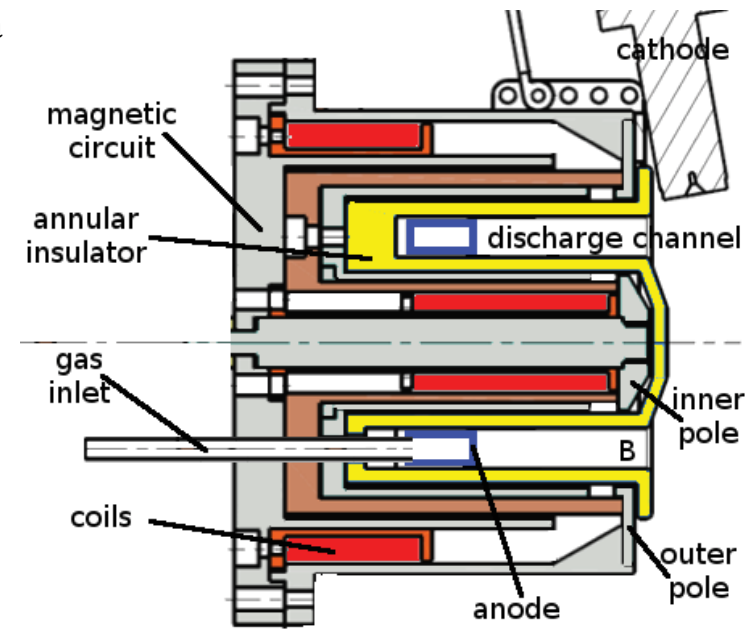

$\mathrm{b}$

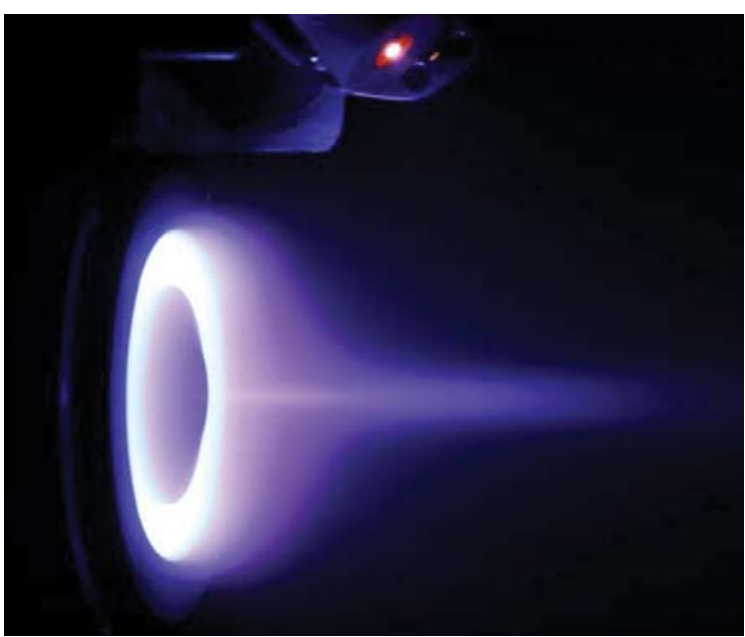

Fig. 1. KLIMT 2nd version: (a) cross section - out of the scale, (b) as operating with krypton in PlaNS laboratory.

with xenon Hall effect thruster which increased the craft speed by $\Delta V \approx 3.8 \mathrm{~km} / \mathrm{s}$. It resulted in entering a lunar orbit in 2004. That successful mission lasted till 2006 [3].

The main goal of the KLIMT project was to figure out if the efficiency of a relatively small HET that is supplied with krypton is comparable with the efficiency produced when xenon propellant is used, and if krypton could indeed be considered as a cost-effective alternative for fueling Hall thrusters of the given size and power class. At the project guidelines formulation phase, it was accepted that the gradually improved implementation of the new engine should be a laboratory/research model of modular structure aimed at the selection of the most efficient configuration. However, while designing the new thruster, several tasks like magnetic field optimization and efficient heat-load handling had to be addressed, despite the fact that KLIMT's architecture has relied on the well-known idea of Hall effect thruster (as described below) $[1,4,5]$. In case of KLIMT, the optimization consisted of numerical modelling, as well as series of experiments. Accordingly, three subsequent prototype versions of KLIMT have been designed and tested to improve the final implementation and to complete the project goals. The relevant test campaigns were arranged in the ESA Propulsion Laboratory (EPL) of ESTEC (Noordwijk, the Netherlands), as well as in the IPPLM's own Laboratory for Satellite Plasma Thrusters (PlaNS).

In most of HETs, the plasma discharge is ignited inside the annular dielectric channel. The anode, which is usually a distributor of neutral gas, is placed at the bottom of this channel. The cathode is placed outside the channel, close to its open end which makes the thruster exit plane (see Fig. 1). The electrodes are biased with DC voltage (typically of $\sim 300 \mathrm{~V}$ ). The set-up axial electric field $\mathbf{E}$ drives the electrons emitted by the cathode towards the channel. In the vicinity of its exit plane, the electrons encounter radial magnetic field $\mathbf{B}$ produced by the electromagnets. The field $\mathbf{B}$ is strong enough to trap the electrons prior to entering the anode. The magnetized electrons drift azimuthally in crossed $\mathbf{E}$ and $\mathbf{B}$ fields, inducing a circular Hall current [5] close to the thruster exit plane. The neutral particles of gas ejected through the anode are effectively ionized in collisions with the drifting electrons, which due to the impact with atoms and channel walls diffuse towards the anode. The axial electric field accelerates ions downwards the thruster channel, producing thrust. The expelled ions capture additional electrons emitted by the cathode, keeping the thruster electrically neutral. The effect of magnetic field on ion trajectories is negligible.

KLIMT was assumed to be a $\sim 500 \mathrm{~W}$ class Hall thruster with elongated acceleration/ionization zone [4] and concentric magnetic coils (external with respect to the internal). The radial scaling down [6-8] relevant to the state-of-the-art SPT100 model design and photographic scaling of its magnetic field topography was applied. Nevertheless, the maximum value of magnetic induction $B_{\max }$ in the channel was increased up to $\sim 0.025 \mathrm{~T}$ to better satisfy $R_{\mathrm{L}}<<\left(b_{c h}\right.$ and $L_{i}$ ) relation, where $R_{\mathrm{L}}$ is the Larmor radius of electrons, $b_{c h}$ is the discharge channel width and $L_{i}$ is the ionization length. For more details on the thruster design guidelines and their implementation, the reader is kindly refereed to our previous papers [9, 10]. Just for clarity, the basic KLIMT's parameters are summarized in Table 1.

It was demonstrated that Hall thruster can operate being fueled with various gases $[11,12]$. However, xenon is considered to be an almost ideal propellant that provides high HET's efficiency. It is due to this noble gas high ionization cross section and atomic mass (131.3 $\mathrm{u})$, as well as low ionization energy $(12.13 \mathrm{eV})$. Moreover, xenon is chemically neutral and does not contaminate any surface. These attributes make xenon a great EP propellant, but unfortunately, its occurrence in the Earth's atmosphere is very low, which results in its extremely high price. Krypton's physical characteristics (lower ionization cross section, ionization potential of $13.5 \mathrm{eV}$ and

Table 1. KLIMT's parameters

\begin{tabular}{lc}
\hline \multicolumn{1}{c}{ Geometry } & Operating parameters \\
\hline$D_{\text {out }}=50 \mathrm{~mm}$ & $U_{D} \leq 500 \mathrm{~V}$ \\
$D_{\text {in }}=34 \mathrm{~mm}$ & $I_{D} \leq 2.0 \mathrm{~A}$ \\
$b_{c h}=8 \mathrm{~mm}$ & $P_{D} \leq 0.5 \mathrm{~kW}$ \\
$L_{c h}=12-35 \mathrm{~mm}$ & $B_{0}=150-250 \mathrm{Gs}, d m / d t \leq 1.5 \mathrm{mg} / \mathrm{s}$ \\
\hline
\end{tabular}



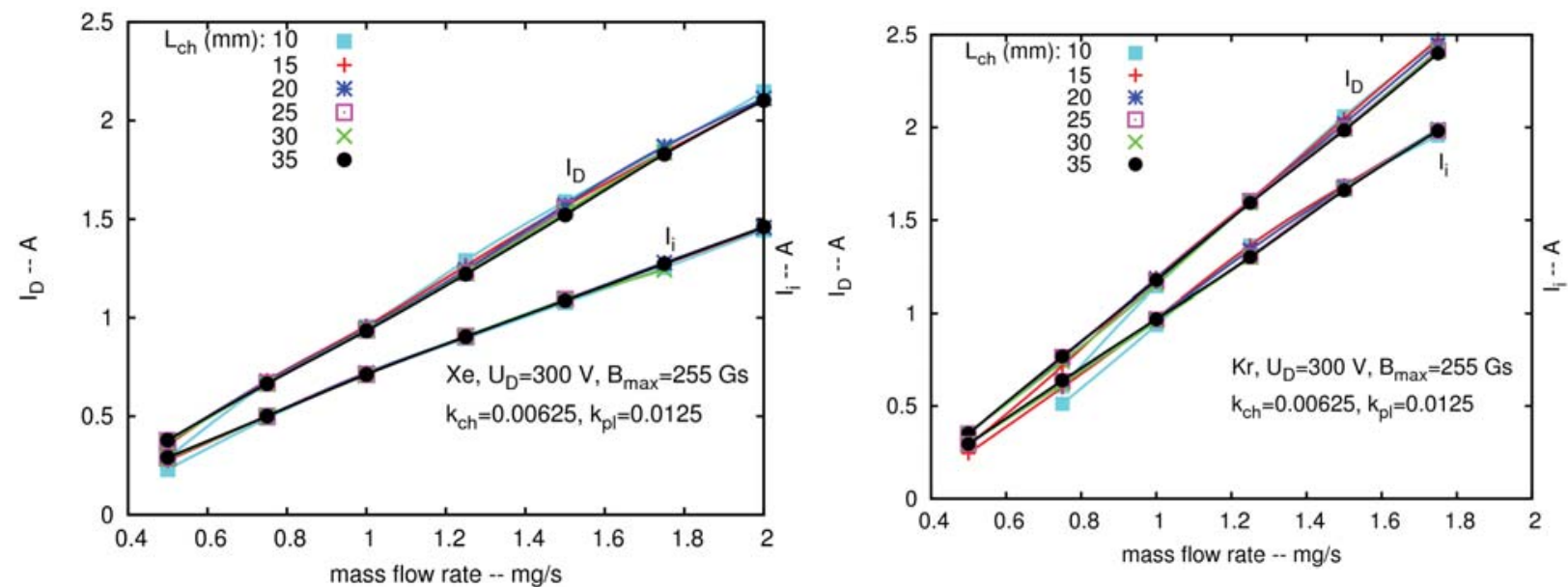

Fig. 2. Comparison of the discharge current $I_{D}$ for xenon and krypton propellants as calculated with HETMAn simulating tool. Ion current fraction $I_{i}$ is also depicted. Calculations are parametrized with the discharge channel length $L_{c h}$.

atomic mass of $83.8 \mathrm{u}$ ) presumed it to be less favourable. Nevertheless, krypton has one advantage - its cost per mass unit is about 5 times lower than that of xenon; and, while discussing the space mission economy, this fact may be the deciding factor to use this slightly less efficient propellant [13].

\section{Heat problem}

Even the early studies support the general idea that krypton HET performance could be on-par with the xenon thruster, if the mass flow rate of $\mathrm{Kr}$ is kept at the same or increased level as compared to Xe [14, 15]. The increased number density of krypton in the discharge channel is able to compensate for its low ionization cross section and improve the mass utilization. However, the discharge current and power also increase leading to the growth in the thruster heat loads. It is illustrated in Fig. 2, in which the numerically calculated values of discharge and ion currents are depicted for both gases. The simulations were performed with the use of the HETMAn 1D hydrodynamic code [16] applied for KLIMT performance estimation [17]. Similar results are observed in the experiments [18]. It is worthwhile to note that according to our calculations, the effect of the channel length is of secondary importance, if the mass flow rate is big enough (see Fig. 2).

In the small krypton HET, the increased heat loads are due not only to the choice of the propellant, but also to scaling down the thruster. In HET, without the active cooling system, the only way to evacuate the excessive Joule heating is radiation. However, firstly the heat energy released inside the thruster has to be transferred to the radiating surfaces. Providing this transfer effectively was one of the main challenges for the KLIMT's design. To assess the increased heat loads and thermal behaviour of each new version, extensive calculations of the temperature field inside the thruster were performed $[9,18]$ prior to the manufacturing of individual parts. The expected temperature distribution in the KLIMT's body was modelled with two simulating tools - heat module of the public domain FEMM code (Finite Element Method Magnetics) [19] and the CRATHER code (un Code de ConductionRAdiation THERmique) [20].

The inner structure of the KLIMT's body consists of concentric elements fixed to the thruster bottom (see Fig. 3) which is linked to the radiator. A reliable thermal interface between these elements and the bottom must be assured to set up a heat sink and provide for efficient cooling. Additionally, for the inner radiative transfer improvement, the effect of a vacuum flask that results between concentric surfaces should be minimized. In simulations, heat transfer due to conduction as well as radiation was considered. However, while in the CRATHER code, radiation between inner surfaces is fully supported (with correctly calculated relevant view factors), the FEMM code allows only for setting radiative boundary conditions (at the external surfaces). On the other hand, in FEMM, the heat conduction is addressed more consistently than in CRATHER. That is why to model the internal radiative transfer within the FEMM, a virtual material between radiating surfaces was introduced as mentioned in our previous paper [9].

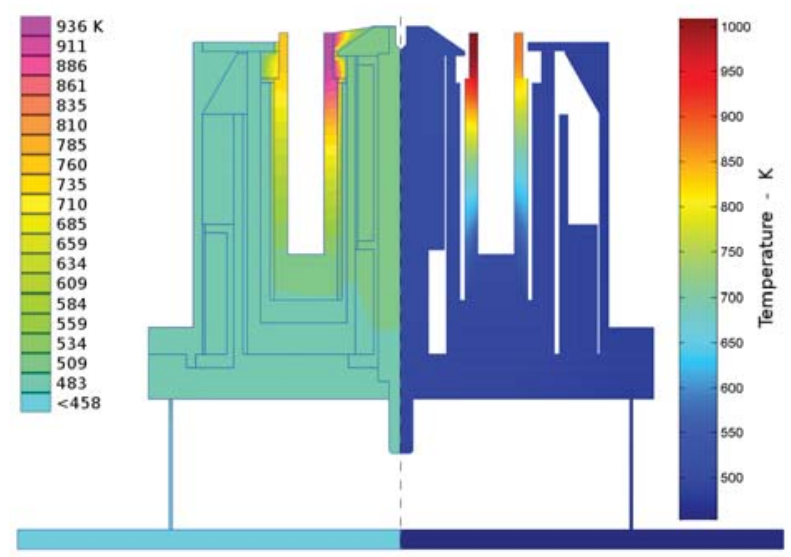

Fig. 3. Comparison of the temperature distributions as calculated with FEMM (the left profile) and CRATHER (the right profile) codes. It was assumed that heat source (plasma) was uniformly distributed at the last $10 \mathrm{~mm}$ of the inner and outer surfaces of the discharge channel. Please note the temperature distribution in the artificial material filling the empty cavities. 
Let us assume that a cylinder of radius $r_{1}$, emissivity $\varepsilon_{1}$ and temperature $T_{1}$ generates heat flux $q_{12}$ towards the concentric cylinder of radius $r_{2}$, emissivity $\varepsilon_{2}$ and temperature $T_{2}$. Assuming that both cylinder lengths $L>>r_{1}$ and $r_{2}=r_{1}(1+\delta)$, where $\delta<<1$, which is well satisfied inside the KLIMT body, the view factor of the infinite cylinders can be used and with a good accuracy the well-known formula reads [21]:

$$
q_{12}=\sigma \frac{T_{1}^{4}-T_{2}^{4}}{\frac{1}{\varepsilon_{1}}+\left(\frac{1}{\varepsilon_{2}}-1\right) \frac{r_{1}}{r_{2}}}
$$

where $\sigma$ is Stefan-Boltzmann constant.

Applying now Fourier law in cylindrical symmetry, heat conductivity $\lambda(T)$ of that virtual material can be deduced from the equation:

$$
q_{12} r_{1}=-\lambda(T) r d T / d r
$$

The simple integration results in the formula:

$$
q_{12} r_{1} \ln \frac{r_{2}}{r_{1}}=S\left(T_{1}\right)-S\left(T_{2}\right)
$$

where $S(T)=\int_{T_{0}}^{T} \lambda(\tau) d \tau$

is the heat potential for which $T_{0}$ is an arbitrary reference temperature. Finally:

$$
\lambda(T)=\frac{4 \sigma T^{3} r_{1} \ln \frac{r_{2}}{r_{1}}}{\frac{1}{\varepsilon_{1}}+\left(\frac{1}{\varepsilon_{2}}-1\right) \frac{r_{1}}{r_{2}}}
$$

Similar calculations can be done for $T_{2}>T_{1}$ and the parallel flat surfaces using appropriate view factors and the problem symmetry. The relevant non-linear heat conductivity 'coefficients' were calculated for the inner KLIMT's cavities and added to the FEMM's database as new materials. This method has been applied for each KLIMT's instance since the heat loads assessment of the first version [9]. An

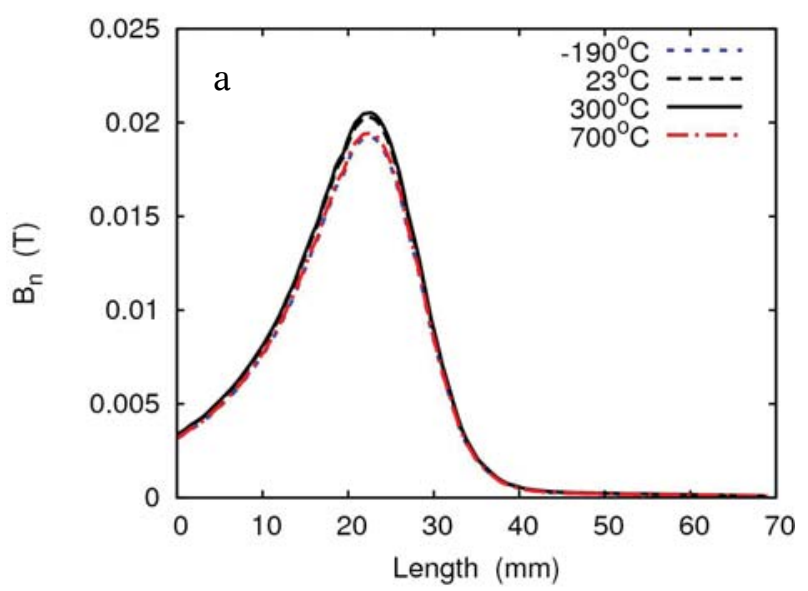

exemplary temperature distribution, as calculated with FEMM and CRATHER for the first prototype, is reproduced in Fig. 3. The agreement of both calculations is satisfactory. The results indicate that the temperature of the most sensitive elements of magnetic circuit is lower than Curie value. Consequently, the actual magnetic field distribution of the operating thruster should be close to the assumed during the design phase. It was verified when $\mathbf{B}$ field distribution was modelled with FEMM, applying the temperature dependent magnetization curves (for the hottest elements of the magnetic circuit, i.e., for the poles) (see Fig. 4). Nevertheless, for all-iron magnetic circuit, the thermal degradation of the magnetic field distribution may be significant and manifest at lower temperature than for magnetic circuit with elements made of FeCo alloy.

Accordingly, in the heat loads modelling, the temperature of the most sensitive parts of thruster, that is windings of the magnetic coils (made of Kulgrid 28 high temperature wire [24]), should not exceed in experiment the temperature limit which is of $\sim 500^{\circ} \mathrm{C}$. However, in practice, the magnetic coils of the first and second prototypes got extremely high temperature before the thruster reached thermal stability. The issue was correctly addressed and solved in the third version of thruster.

\section{Test results}

The lessons learned from each measurement campaign provided a feedback for the next design phase. The results of tests that were performed in both laboratories (EPL and PlaNS) confirmed the correctness of the main assumptions to the new thruster architecture. However, it clearly appeared that the design of some elements of the magnetic circuit should be corrected. To make the magnetic circuit thermally stable and resistant to the increased heat loads, in the second version, its hottest elements were replaced by parts made of FeCo alloy [18] with Curie temperature about $200 \mathrm{~K}$ higher than the soft iron. In the third version, all the thermal interfaces were

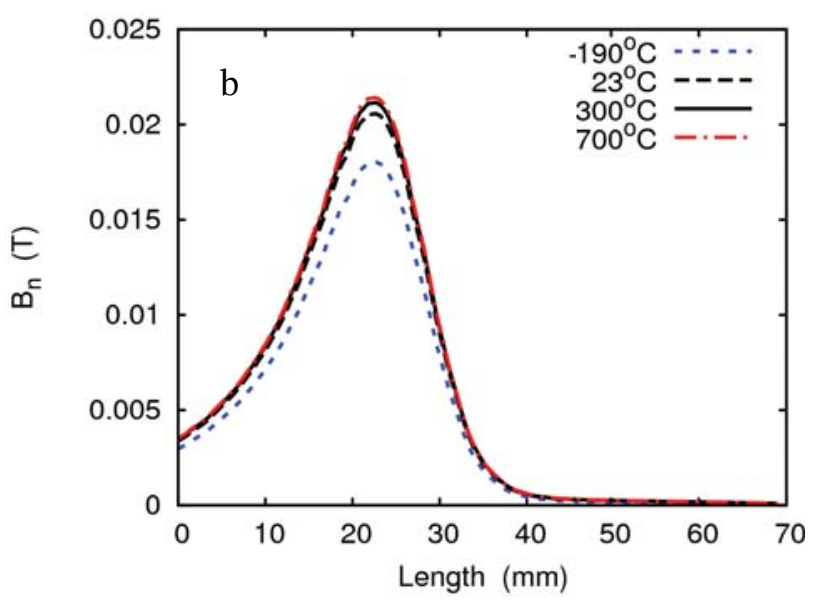

Fig. 4. Axial component at $\mathbf{B}$ field at the axis of discharge channel as calculated with FEMM for $I_{\text {in }}=0.5 \mathrm{~A}$ and $I_{\text {out }}=$ 0.5 A coil currents: (a) for all iron magnetic circuit, (b) for magnetic circuit with poles and inner core made of FeCo alloy. The temperature of the hottest parts (poles) of the circuit is indicated in the figure legends. Magnetization curves for pure iron and FeCo alloy were calculated relying on analytical approximation according to [22, 23]. 


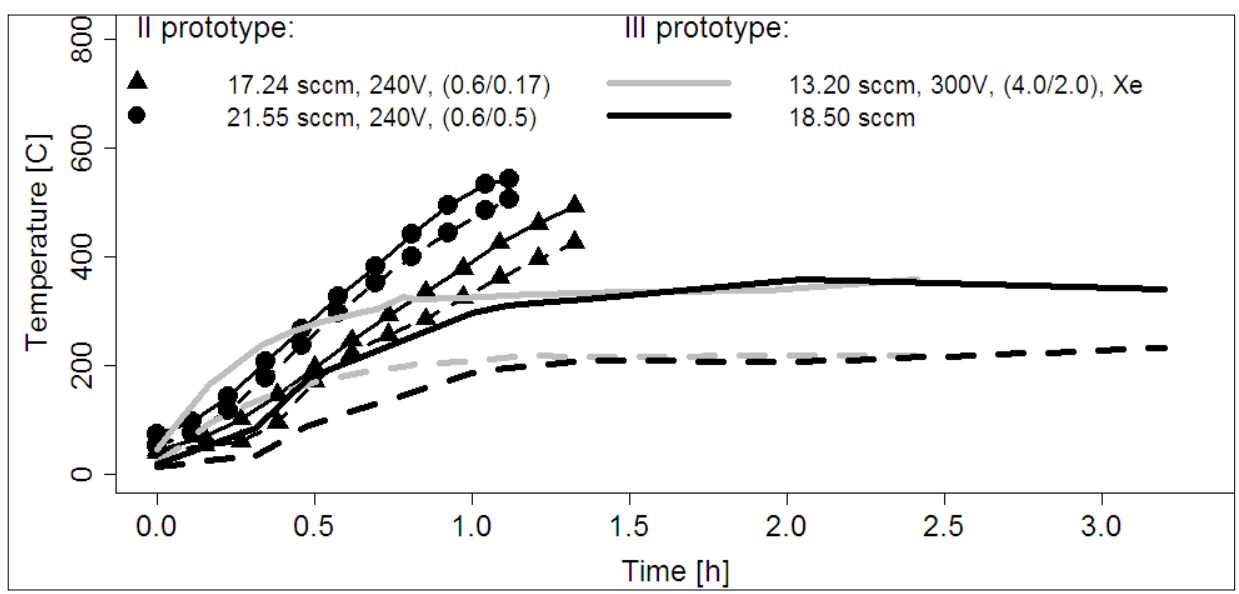

Fig. 5. Inner and outer (dashed) coil temperature vs. operation time for the last two prototypes.

refined, while the radiated surfaces blackened to increase effectiveness of cooling by radiation and to reduce the vacuum flash effect. New solution for the internal heat guides and screens (thermal and magnetic ones) was implemented. These improvements resulted in the new structure of the magnetic circuit, however, the topology of $\mathbf{B}$ field was preserved [18]. The effectiveness of the modified thermal layout was confirmed numerically. Due to the introduced modifications, the outer diameter of the thruster and its length (as well as the length of BN channel) were both reduced by $\sim 1 \mathrm{~cm}$, decreasing the total weight of the thruster. Finally, the third prototype turned out to be thermally stable, and all its characteristics were measured at the well-established equilibrium temperature.

Nevertheless, the thermal equilibrium of both the prototype versions could not be gained within the safety limits of the coils. Therefore, to make the interpretations of early tests more straightforward, the measurements were performed with the temperature of the thruster strictly controlled. The inner and outer coil temperatures were determined relying on their resistivity variation during the operation. A linear temperature relation for specific resistance of the Klugrid 28 wire was assumed based on the producer data-sheet [24]. The recording coil current $I_{c}$ and voltage $U_{c}$, knowing the coil resistivity $R_{\text {ref }}$ in reference to the temperature $T_{\text {ref }}$, as well as cables resistivity $R_{\text {cab }}$, one can calculate the coil temperature $T_{c}$ with the simple formula:

$$
T_{c}=\frac{1}{\alpha}\left(\frac{U_{c} / I_{c}-R_{\mathrm{cab}}}{R_{\mathrm{ref}}-R_{\mathrm{cab}}}-1\right)+T_{\mathrm{lab}}
$$

where $T_{\text {lab }}$ is the temperature of laboratory and $\alpha$ is the temperature coefficient of resistance. $T_{\text {lab }}$, $R_{\text {cab }}$ and $\alpha$ were assumed to be constant during the measurements.

By its physics, the HET continues to operate as long as it is supplied with voltage and propellant. However, KLIMT was intentionally switched off always when the inner coil temperature came too close to the limit of $500^{\circ} \mathrm{C}$ [10].

Approaching thermal equilibrium by the third prototype for krypton and xenon is illustrated in Fig. 5. For comparison, the temperature variations of second prototype during its operation are also de- picted. The second prototype was operated only with krypton. Please note that for krypton, the flow rate of $21.6 \mathrm{sccm}$ temperature increases much faster than for $17.2 \mathrm{sccm}$, despite the fact that discharge voltage is kept at the same level. It is obvious, since with the increased krypton number density, the discharge current and power also increases resulting in the growth of Joule heating. The second prototype had to be switched off prior to getting damaged due to overheating, usually after $1-1.5$ hour of operation.

Testing the thruster performance prior to gaining thermal equilibrium, the impact of temperature had to be examined. Studying KLIMT's performance in PlaNs laboratory, we were not able to measure the thrust in an absolute manner (such measurements were performed for KLIMT only in EPL). Instead, we captured the plasma jet with a graphite target that was placed at the thrust balance. The resulting force $F$ produced by the momentum flux was assumed to be proportional to the thrust. The method is somehow similar to that described in paper [25]. The variations of $F$ vs. inner coil temperature is reproduced in Fig. 6. Additionally, the discharge current is depicted in the same figure. It is clearly seen that both characteristics slowly decrease with the growth of thruster temperature. Permanent monitoring of temperature allowed us for consistent comparison of thruster characteristics when it was operating even in transient states.

To better prepare the third measurement campaign in EPL, an introductory session in PlaNS

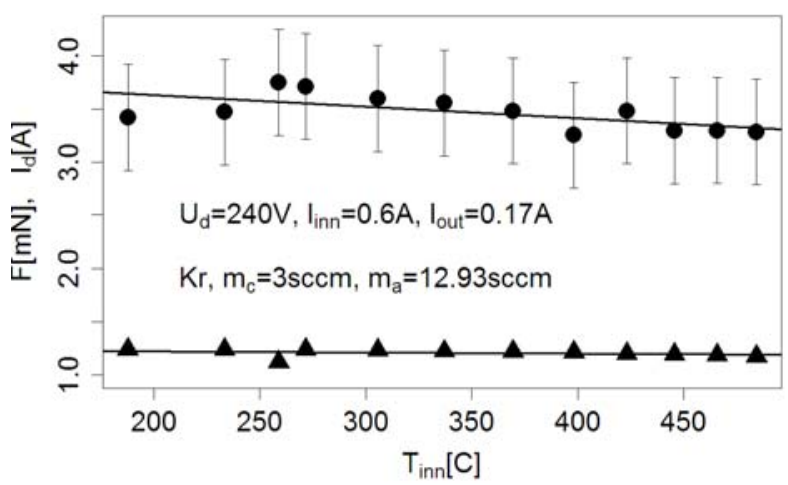

Fig. 6. Momentum flux (denoted with •) as captured by graphite target placed at the thrust balance and discharge current $(\boldsymbol{\Lambda})$ vs. temperature of inner coil. 


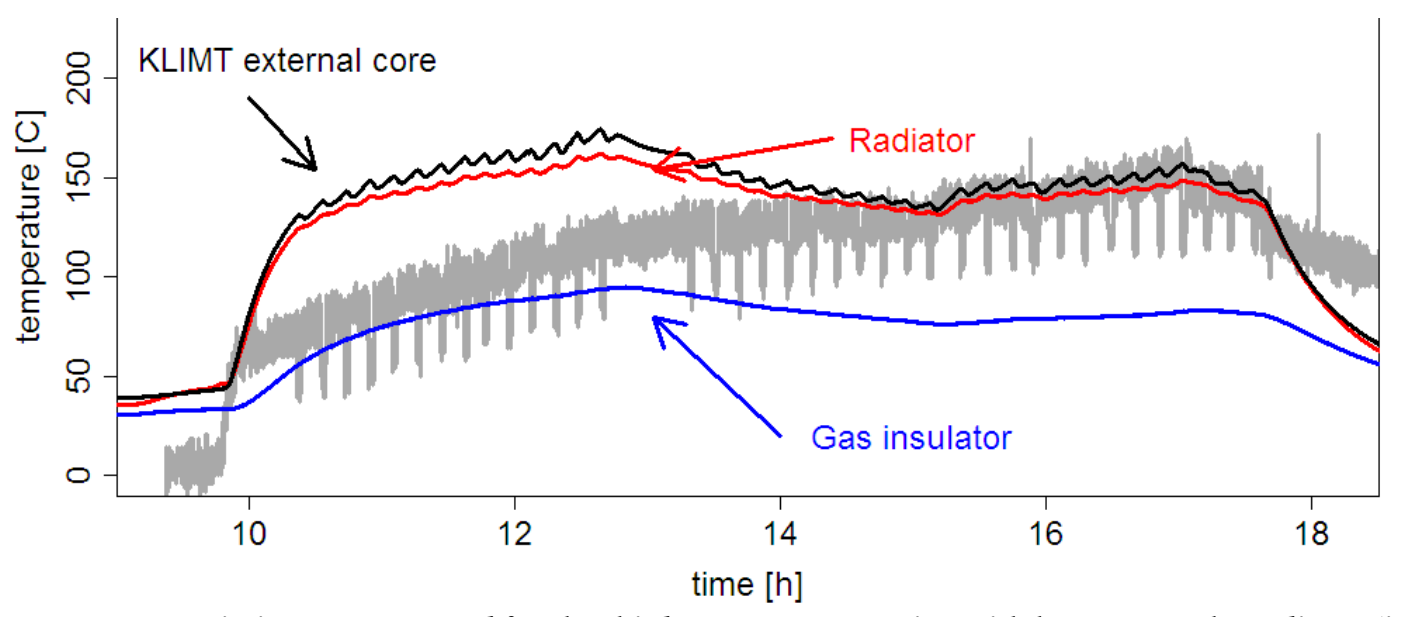

Fig. 7. Temperature variations as measured for the third prototype operating with krypton: at the radiator (in red), at the external core of the magnetic circuit (in black) and the gas insulator of the anode line (in blue). The thrust balance record is added (in gray, out of the scale) to illustrate switching on-off cycles.

laboratory was arranged with the aim at preliminary characterization of the final KLIMT's version. When probing its operational envelope, more than two hundred set-points were examined. Accordingly, the mass flow rates, discharge voltage and coil currents were varied in the wide range, while relevant discharge currents (time dependent and averaged), as well as momentum flux to the graphite target were recorded. The preliminary measurements were repeated in EPL, however, only for precisely the selected set of operating conditions, at which the absolute thrust values were recorded for both gases. Additionally, beam divergence was measured, but only for krypton [18].

In EPL, the thruster thermal state was monitored relying not only on the resistivity variation of both coils (Eq. (5)) but also by means of K-type thermocouples. They were used for temperature measurement of the thruster radiator, external core of magnetic circuit and gas insulator in the anode line. The results as recorded during the one-day experiment are presented in Fig. 7. In the same figure, the relevant record of thrust balance was also reproduced. It illustrates the duration of switching on-off cycles which were applied to measure the thrust magnitude at the background of the long term drift of the thrust balance. Fast temperature variations are obviously coherent with the operating cycles of the thruster. Slow temperature changes are relevant to systematic variations of the operating conditions that were intentionally modified during the measurements. It is worth noting that the temperature of the external parts of the thruster (in its thermally stable version) was at least two times lower than the temperature of the inner coil (compare Fig. 5 and Fig. 6), which in turn was always lower at least by $100^{\circ} \mathrm{C}$ than the safety limit.

\section{Conclusions}

The aim of Hall effect Krypton Large IMpulse Thruster (KLIMT) project was the assessment of krypton as a propellant when used alternatively to xenon. The new thruster has been designed from the ground up resulting in manufacturing of the three prototype versions. The results of the thruster performance comparative tests were presented elsewhere [18]. Here, we focused on thermal stability of the new design. The presented test results show that increased heat loads, which are an inherent feature of the krypton thruster, have been correctly addressed in the KLIMT's final version. It means that the materials as selected for the magnetic circuit, heat guides, coils and insulators appeared to be chosen properly. The effectiveness of heat excess evacuation from the thruster interior to the dedicated radiator was demonstrated (by experimental characterization). The improvement of thermal interfaces provided for thruster operation within the safety limits even of the most sensitive materials. The final version turned out to be thermally stable for all the given operating conditions and worked with krypton reliably as long as it was required. One can conclude that krypton could be seriously considered as an alternative propellant for HET.

Acknowledgments. The authors would like to thank Dr Zbigniew Płochocki for a lot of discussions on radiative heat transfer problem and formulation it in terms of heat potential. The project KLIMT has been supported by ESA since March 2013 in the frame of the second PECS call (ESA contract No. 4000107746/13/NL/ KLM). The development of HETMAn has been partly funded by the European Commission (FP7 'HiPER' project, No. 218859) and has received financial support from the Foundation for Polish Science in the year 2011.

The 13th edition of the Kudowa Summer School "Towards Fusion Energy", held in Kudowa Zdrój, Poland on 13-17 June 2016, was supported by the Polish Physical Society as well as the Polish Ministry of Science and Higher Education.

\section{References}

1. Morozov, A. I., \& Savelyev, V. V. (2000). Fundamentals of stationary plasma thruster theory. Rev. Plasma Phys., 21, 203-391. 
2. Kim, V., Popov, G., Arkhipov, B., Murashko, V., Gorshkov, O., Koroteyev, A., Garkusha, V., Semenkin, A., \& Tverdokhlebov, S. (2001). Electric propulsion activity in Russia. In Proceedings of the 27th International Electric Propulsion Conference, Pasadena, CA, USA (paper 05).

3. European Space Agency. (2009, August 1). SMART-1 overview. Retrieved from www.esa.int/Our_Activities/ Space Science/SMART-12.

4. Zhurin, V. V., Kaufman, H. R., \& Robinson, R. S. (1999). Physics of closed drift thrusters. Plasma Sources Sci. Technol., 8, R1-R20.

5. Goebel, D. M., \& Katz, I. (2008). Fundamentals of electric propulsion: Ion and Hall Thrusters. Hoboken, New Jersey: Wiley.

6. Ahedo, E., \& Gallardo, J. M. (2003). Scaling down Hall thrusters. In Proceedings of the 28th International Electric Propulsion Conference, Toulouse, France (paper 104).

7. Dannenmayer, K., \& Mazouffre, S. (2011). Elementary scaling relations for Hall Effect Thrusters. J. Propul. Power, 27, 236-245.

8. Shagayda, A. A. (2013). On scaling of Hall Effect Thrusters. In Proceedings of the 33rd International Electric Propulsion Conference, Washington, D.C., USA (paper 56).

9. Kurzyna, J., \& Daniłko, D. (2011). IPPLM Hall Effect Thruster - design guidelines and preliminary tests. In Proceedings of the 32nd International Electric Propulsion Conference, Wiesbaden, Germany (paper 221).

10. Kurzyna, J., Barral, S., Daniłko, D., Miedzik, J., Bulit, A., \& Dannenmayer, K. (2014). First tests of the KLIMT Thruster with Xenon propellant at the ESA Propulsion Laboratory, Space Propulsion Conference, Cologne, Germany.

11. Makela, J. M., Washeleski, R. L., Massey, D. R., King, L. B., \& Hopkins M. A. (2009). Development of a magnesium and zinc Hall-Effect Thruster. In Proceedings of the 31st International Electric Propulsion Conference, Ann Arbor, Michigan, USA (paper 107).

12. Scharfe, D. B. (2009). Alternative Hall thruster propellants krypton and bismuth: Simulated performance and characterization. Ph.D. thesis, Stanford University.

13. Nakles, M. R., William Jr., A. H., Delgado, J. J., \& Corey R. L. (2011). A performance comparison of xenon and krypton propellant on an SPT-100 Hall Thruster. In Proceedings of the 32nd International
Electric Propulsion Conference, Wiesbaden, Germany (paper 003).

14. Kim, V., Popov, G., Kozlov, V., Skrylnikov, A., \& Grdlichko, D. (2001). Investigation of SPT performance and particularities of its operation with $\mathrm{Kr}$ and $\mathrm{Kr} / \mathrm{Xe}$ mixtures. In Proceedings of the 27th International Electric Propulsion Conference, Pasadena, CA, USA (paper 065).

15. Linnell, J. A., \& Gallimore, A. D. (2005). Efficiency analysis of a Hall Thruster operating with krypton and xenon. In Proceedings of the 41st AIAA/ASME/SAE/ ASEE Joint Propulsion Conference and Exhibition, Tucson, Arizona, USA (paper 3683).

16. CORDIS. (2013). Final report to FP7 European project HiPER (High Power Electric propulsion: A roadmap for the future). Contract no. 218859. EC Community Research and Development Information Service.

17. Kurzyna, J. (2014). Numerical investigation of the Krypton Large IMpulse Thruster. Phys. Scripta, T161, 014051(4 pp.).

18. Kurzyna, J., Szelecka, A., Daniłko, D., Barral, S., Dannenmayer, K., Bosch Borras, E., \& Schönherr, T. (2016). Testing KLIMT prototypes at IPPLM and ESA Propulsion Laboratories. In Proceedings of Space Propulsion, Rome, Italy.

19. Meeker, D. C. (2010). Finite element method magnetics. Version 4.2 Nov. Build, http://www.femm.info.

20. Barral, S., \& Brayer, C. (1997). CRATHER: un code de Conduction-RAdiation THERmique. National Center for Scientific Research, France.

21. Çengel, Y. A. (2002). Heat transfer - a practical approach (2nd ed.). Boston: McGraw Hill.

22. Włodarski, Z. (2006). Analytical description of magnetization curves. Phys. B-Condens. Matter, 373, 323-327.

23. Włodarski, Z., \& Włodarska, J. (1998). Analytical approximation of the dependence of magnetic material properties on temperature. COMPEL, 402-406.

24. Ceramawire. (2011). Ceramawire High Temperature Magnet Wire Technical Specs. http://www.ceramawire.com/technical-information.shtml\#2.

25. Longmier, B. W., Reid, B. M., Gallimore, A. D., ChangDíaz, F. R., Squire, J. P., Glover, T. W., Chavers, G., \& Bering III, E. A. (2009). Validating a plasma momentum flux sensor to an inverted pendulum thrust stand. J. Propul. Power, 25, 746-752. 